NASA Technical Memprandum 87581

\title{
A MIXED PSEUDOSPECTRAL/FINITE DIFFERENCE METHOD FOR A THERMALLY DRIVEN FLUID IN A NONUNIFORM GRAVITATIONAL FIELD
}

MICHELE G. MACARAEG

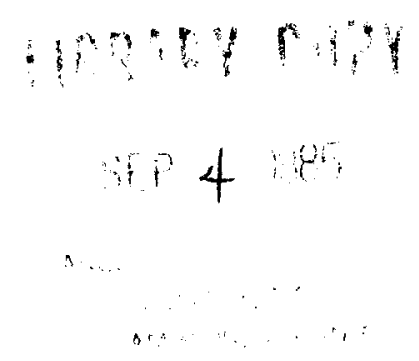

JULY 1985

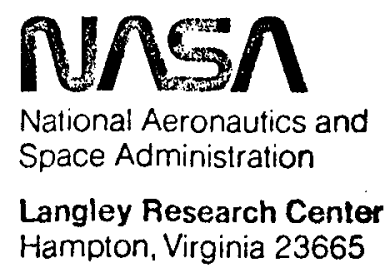





\section{Abstract}

A numerical study of the steady, axisymmetric flow in a heated, rotating spherical shell is conducted to model the Atmospheric General Circulation Experiment (AGCE) proposed to run aboard a later shuttle mission. The AGCE will consist of concentric rotating spheres confining a dielectric fluid. By imposing a dielectric field across the fluid a radial body force will be created. The numerical solution technique is based on the incompressible Navierstokes equations. In the method a pseudospectral technique is used in the latitudinal direction, and a second-order accurate finite difference scheme discretizes time and radial derivatives. This paper discusses the development and performance of this numerical scheme for the AGCE which has been modelled in the past only by pure FD formulations. In addition, previous models have not investigated the effect of using a dielectric force to simulate terrestrial gravity. The effect of this dielectric force on the flow field is investigated as well as a parameter study of varying rotation rates and boundary temperatures. Among the effects noted are the production of larger velocities and enhanced reversals of radial temperature gradients for a body force generated by the electric field.

\section{Background}

Since as early as the nineteenth century there have been experimental efforts aimed at better understanding the earth's atmosphere. Until the 1950's, however, these efforts were qualitative in nature. It was found that experiments involving a fluid confined between two concentric, rotating cylinders could be systematically studied. Experiments performed by Hide 1 in 1953, using a cylindrical annulus, resulted in the discovery of a meandering jet which greatiy resembled the earth's jet stream. In 1965 , Fowlis and Hide 2 quantitatively produced" a flow regime diagram by careful measurements taken from the annulus experiments. Figure 1 , is the regime diagram adapted from Fowlis and Hide. The ordinate is a thermal Rossby number, and the abscissa a Taylor number, both of which are given in equations (1) and (2):

$$
\begin{aligned}
& R o_{T}=\frac{\text { agstd }}{\Omega^{2}\left(r_{0}-r_{i}\right)^{2}} \\
& T a=\frac{4 \Omega^{2}\left(r_{0}-r_{i}\right)^{5}}{v^{2} d}
\end{aligned}
$$

where $g$ is the acceleration due to gravity, $a$ is the thermal expansivity, $\Delta T$ is the horizontal temperature difference, $d$ is the annulus height, ( $\left.r_{0}-r_{i}\right)$ is the radial thickness, $B$ the rotation rate, and $u$ the kinematic viscosity. The knee-shaped curve in figure 1. referred to as the critical curve, separates the axisymmetric flow regime from the wave-like flow (nonaxisymmetric regime).

The flow in the nonaxisymmetric regime is an analogue of the synoptic scale motion of a planetary atmosphere. The annulus experiments have therefore guided studies concerning the predictability or periodicity of the atmosphere. Scaling of the governing equations based on large scale features of the atmosphere and laboratory experiments leads to the same set of approximate equations for both systems. Thus. the experiments are models of the synoptic-scale atmospheric flow.

Since analytical solutions are not obtainable due to the strong nonlinearity of the governing equations, numerical modelling has served to enhance the theory and provide a quantitative comparison with experiment. Although models are becoming increasingly more complex, substantial advances in atmospheric studies have come from simple models which have isolated essential mechanisms concerning the earth's general circulation. It was observed by Lorenz 3 that a steady axisymmetric solution to the governing equations exists everywhere in parameter space. The reason for the nonaxisymmetric regimes is the instability of the axisymmetric flow to infinitessimal (nonaxisymmetric) pertubations. The first step, therefore, in determining whether a given flow is stable is to calculate the steady, axisymnetric states. If a given axisymmetric solution is unstable to perturbations of any wavelength, then it might not be observed in a physical situation. Axisymmetric states for the thermally driven motion of a fluid contained in a rotating cylindrical annulus were numerically investigated by Williams 4,5 . Axisymmetric states were thoroughly studied, but a variety of points in parameter space were not considered. Miller and Gall 6 used a linear model to predict a transition curve very close to that observed in the laboratory. 
An obvious disadvantage of cylindrical models of the earth's atmosphere is the geometrical difference. However, experimental studies done in a spherical geometry on earth cannot generate a radial body force strong enough to compete with terrestrial gravity. Such an experiment needs the near zero gravity of Spacelab to generate a sufficiently dominate radial body force.

The numerical model in this study will serve as an aid in constructing a regime diagram for a spherical geometry, analogous to the cylindrical annulus case (Figure 1.) The spherical regime diagram is also most essential for the AGCE, since knowing the location of the axisymmetric and nonaxisymmetric flow regimes is necessary for the design of the AGCE instrument.

In addition to the need for a spherical regime diagram for AGCE, it is desirable to know the effect of the radial variation of the dielectric gravitational field. The radial force created by imposing an electric field on the dielectric fluid is inversely proportional to the fifth power of the radius\%. On earth, the gravitational force is inversely proportional to the square of the radius. The effect of varying this power is needed to determine how capable the dielectric force is in simulating terrestrial gravity.

\section{I. Physical Mode?}

A schematic of the experimental set-up is shown in Figure 2. A dielectric flutd will be confined in a portion of the gap between the spheres by placing solid walls in the spherical domain. The annulus is subdivided to provide room for instrumentation. Temperature profiles on the spheres will simulate the equator to pole distribution and establish the large-scale vertical stability (warmer outer sphere) of the atmosphere. The gravitational body force on the atmosphere is simulated by application of an electric field across the gap which generates a radial body force in the dielectric fluid. In order to be radial, this force requires the zero gravity environment of space, since the earth's gravitational force would greatly distort the simulated gravitational force field.

\section{The Numerical Model}

The right-handed coordinate system used in the model is rotating with the apparatus at the constant angular velocity $\Omega$. Figure 3 . depicts this system with $r$ representing the radius, $\theta$ the co-latitude, and the azimuthal angle. The governing equations are solved in a vorticity/ stream function formulation for a Boussinesq fluid. The solid body rotation rate, $\Omega$, nondimensionalizes time. The characteristic radial velocity is taken as the solid body rotation rate times the gap width--all other length scales are nondimensionalized by the outer sphere radius. A nondimensional temperature is obtained from the difference between the upper and lower temperature extremes on the spherical walls. The nondimensional (unprimed) variables vorticity $(\xi)$, stream function $(\psi)$, and swirl (w) are defined in equations (3) through $(7)$.

$$
\begin{aligned}
& T=\left(T_{U}-T_{L}\right) T^{\prime}+T_{L} \\
& \xi=r_{0}^{\Omega \xi^{\prime}} \\
& \psi=\psi^{\prime} r_{0}^{3} \Omega \\
& \omega=\omega^{\prime} r_{0}^{3 \Omega} \\
& \bar{V}=\bar{V}^{\prime} r_{0} \Omega_{0}
\end{aligned}
$$

The nondimensional momentum and energy equations (where unprimed variables are here nondimensional) are:

$$
\frac{D \xi}{D t}-\frac{2 \xi}{r^{3} \sin \theta}\left(\frac{\partial \psi}{\partial r}-r \cot \theta \frac{\partial \psi}{\partial \theta}\right)-
$$$$
\frac{2 \omega}{r^{3} \sin \theta}\left(\frac{\partial \omega}{\partial \theta}-r \cot \theta \frac{\partial \omega}{\partial r}\right)
$$

$$
=\frac{2 \Omega \sin \theta}{r}\left(\frac{\partial \omega}{\partial \theta}-r \cot \theta \frac{\partial \omega}{\partial r}\right)+
$$

$E K D^{2} \xi+G r E k^{2} \sin \theta\left(\frac{1}{r}\right)^{n} \frac{\partial T}{\partial \theta}$

$\frac{D \omega}{D t}=\frac{-2 \Omega \sin \theta}{r}\left(\frac{\partial \psi}{\partial \theta}-r \cot \theta \frac{\partial \psi}{\partial r}\right)+E k D^{2} \nu$

$\frac{D T}{D t}=\frac{E k}{P r} \nabla^{2} T$

$D^{2} \psi=E$

Boundary conditions appropriate for the AGCE were incorporated, i.e., the no-slip conditions and conducting walls. Details of the numerical model may be found in previous work ${ }^{8}$. A brief summary of the scheme is now given.

As stated earlier the numerical model utilizes a mixed pseudospectral/finite difference (PS/FD) scheme. Due to the presence of the 
latitudinal side walls (see figure 2.), Tschebyshev. polynominals are used as the spectral expansion functions. In addition Tschebyshev collocation methods insure an exponential rate of convergence as opposed to the algebraic rates associated with FD. methods9. It was sufficient to use the pseudospectral formulation in only one direction (latitude) since the computational grid required $1 / 7$ to $1 / 3$ the number of grid points as the pure FD model.

The numerical scheme treats the rotation and advection terms explicity while radial diffusion terms are implicity solved. Pseudospectral derivatives are computed by setting up appropriate matrices for Tschebyshev collocation outside the time iteration process. Latitudinal derivatives are thus computed via a simple matrix multiplication at each time step.

The Poisson equation for stream function was solved in two ways. Initially a pseudo-time derivative was introduced so that at steady state the Poisson equation would be satisfied. During this internal iteration latitudinal derivatives were not updated, which introduced a stabilizing factor in the scheme. This effective "pseudo-time lag" in the Poisson iteration allowed the model to utflize a time step comparable to an implicit formulation in the overall iteration scheme. Alternatively, the matrix corresponding to the Laplacian operator was inverted by a conventional LU decomposition and back solution. Though a fewer number of steps were required in the latter formulation a much more stringent time interval restriction was found necessary.

\section{Numerical Model Application: Varying}

\section{Experimental Parameters}

The main results of an extensive parameter study which varied boundary temperatures and rotation rates for several gravitational force distributions will now follow. The latitudinal side walls were set at $\pi / 4$ and $3 \pi / 4$. The inner and outer sphere radij were set at 5 and $6 \mathrm{~cm}$., respectively. The term governing the force distribution is the buoyancy term of the vorticity equation (Eq. 8 ) as given below:

$$
\operatorname{GrEK}^{2} \sin \theta\left(\frac{1}{r}\right)^{n} \quad \frac{\partial T}{\partial \theta}
$$

In the cases to be discussed, the exponent, $n$, and gravitational acceleration, $g$, were varied under the restriction that the mean gravitational acceleration be maintained at $980 \mathrm{~cm} / \mathrm{s}^{2}$ This was done by calculating a gref as given in (11):

$$
g_{r e f}=980\left(r_{0}-r_{i}\right)\left[\int r_{r_{i}}{\frac{r_{0}}{r}}^{n} d r\right]^{-1}
$$

Cases I through IV, defined in Table 1., allow the study of the effect on the flow field if $G r$ and $E k$ are allowed to vary by four orders of magnitude while maintaining a uniform gravitational field $(n=0)$. Each of these quantities have physical significance. The Grashof number represents the ratio of buoyancy to viscous forces. It appears in the buoyancy term of the vorticity equation numbered 8 . The Ekman number measures the ratio of viscous to Coriolis forces in the flow field. This parameter is found in the vorticity and energy equations. If $\mathrm{Gr} \neq 0$, it couples each of the other equations through the vorticity equation. If $\mathrm{Gr}=0$, the energy equation becomes uncoupled.

Figures 4. through 9. are contour plots of $\psi, w, u, v, T$, and $\xi$ for all four cases. Most noted in the contour plots is the increasing departure from symmetry. In addition, increased meridional transport for increasing $\mathrm{Gr}$ is evident in plots of stream function (Figure 4.) The radial and zonal jets seen in the contours of $w$ (Figure 5.) and $u$ (Figure 6.), respectively, are seen to move northward as buoyancy forces increase (higher Gr). The latitudinal jets associated with $v$ (Figure 7.) become more concentrated towards the spherical wall. Strong thermal boundary layers develop as is evident in temperature contour plots of Case IV, relative to the previous cases, which for Case 1 is essentially a conduction profile (Figure 8.). Vorticity maxima are seen in Figure 9. to shift from the inner radidl wall at mid-latitudes for case I to the wall at $\pi / 4$ and approximately $5.6 \mathrm{~cm}$ for Case IV. These effects are all associated with an increasing buoyancy force which appears to be the dominant factor relative to increasing rotation by the same order of magnitude.

Listed in Table 1. is the product GrEk² calculated for the four cases. This product represents the ratio of buoyancy to Coriolis forces. GrEk ${ }^{2}$ is the smallest in Case IV which has the largest flow field values. The enhancement of advection for decreasing values of GrEk ${ }^{2}$ can be explained as follows. With a radial gravitational field, warm fluid will tend to rise toward the outer sphere. For large $\mathrm{GrEk}^{2}$ numbers, buoyancy forces more effectively keep warm fluid near the outer sphere. If buoyancy forces are weakened, meridional flow will increase to enforce the stable stratification of fluid in the interior.

An interesting study regarding heat transfer rates for the four cases was made by carrying out a heat balance on the domain from $\pi / 4$ to $\pi / 2$ and from $r_{i}$ to $r_{0}$. Ratios of the amount of heat transfer occurring at given boundary over that occurring in pure conduction are 
listed in Table 1. The temperature isotherms of Figure $8 c$. (Case III) and 8d. (Case IV) indicate negative radial thermal gradients around $\theta=$ $5 \pi / 12$ near the lower spherical wall. This is due to the increasing meridional motion for these two cases relative to Cases I and II which carry warm fluid northward as cold fluid sinks towards the equator. This motion acts to decrease the radial thermal gradients near the spherical walls and, for cases of strong meridional motion, to even reverse these gradients. The result is a decrease in the amount of heat transfer occurring at the spherical walls as evidenced in Table 1.

It should be noted that at steady state no net torque is exerted on the fluid. Thus the sum of the viscous stresses exerted by the boundaries on the fluid should vanish. This means that the distribution of easterly and westerly flows at the boundaries should be about equal throughout the fluid volume. Such a trend is seen in the contour plots of azimuthal velocity (Figure 7). However, the distribution of easterlies and westerlies in the atmosphere is different, which is expected since there is no outer boundary as is the case for the AGCE. Time-averaged atmospheric data yield the polar easterlies, the mid-latitude westerlfes and the tropical easterly trade winds. In the AGCE model, contour plots of azimuthal velocity reveal a region of positive zonal flow (westerlies) in the upper half of the domain, while the lower portion, down to the equator shows negative zonal velocity (easterlies). These flows may be studied using conservation principles. If a particle at the equator, having initially no zonal (easterly or westerly) component in its motion, goes toward the polar axis while conserving its angular momentum, it is flung eastward. Conversely, a particle going toward the equator from one of the poles would have a deficit of west-to-east momentum with respect to the earth and would have to curve toward the west. The resulting zonal linear speeds with respect to the earth's surface can be computed by applying the principle of conservation of angular momentum for a unit mass. However, the observed wind speeds in the atmosphere are substantially less than those calculated on the basis of angular momentum conservation. It is believed that in the earth's atmosphere the meridional flow breaks down into smaller circulations or cells.10 Throughout the atmosphere the meridional components are on the average less than one-tenth of the zonal components. It was found in this numerical study that meridional flow values were on the average one-third of the zonal flow. The break-up of the meridional flow into two cells was noted to occur under specific conditions. At approximately the same location where temperature contours indicated a gradient reversal, the stream function contours were found to have two smaller cells in addition to the large cell representing the main meridional circulation. The reversal of the latitudinal gradient of temperature is certain to be a factor in causing these recirculations. It was noted that these recirculations were stronger for values of $n$ equal to 0 and 2 . Higher values of $n$ were also found to increase flow field values. It is possible that these higher values are due to the weakening of these recirculating cells in the meridional flow for the cases of $n$ $=5$. Stream function contours show the appearance of these recirculations which are. seen to be more prevalent for $n=0$ as opposed to $n=2$ and even less pronounced when $n=5$. Stream function contours where rotation was increased from $1 \mathrm{rad} / \mathrm{s}$ to $3 \mathrm{rad} / \mathrm{s}$ showed the appearance of these smaller cells for increased rotation, which also inhibits meridional flow.

\section{Discussion}

Experimentation with the dielectric force ( $n=5$ ) should be observed with the knowledge that differences do exist between the resulting flow field and a flow calculated with a force proportional to $1 / r^{2}$ (the earth's gravitational force). Flow field values will tend to be higher for the dielectric force. The meridional flow's breakup into two smaller cells, which has been observed in the atmosphere and shown numerically for the cases of $n=0$ and $n=2$, will be less pronounced for flows generated with a dielectric force. To compensate for these differences, rotation rates should be at least greater than $1 \mathrm{rad} / \mathrm{s}$ since meridional flow is inhibited by rotation. In addition, boundary temperature differences should not be too extreme so that internal temperature gradients will be weak enough to allow gradient reversals and subsequent recirculations in the meridional flow to occur.

\section{References}

'Hide, R., "Some Experiments on Thermal Convection in a Rotating Liquid," Quarterly Journal of the Royal Meteorological Society, $79: 161,1953$.

${ }^{2}$ Fowlis, W. W. and R. Hide, "Thermal Convection in a Rotating Annulus of Liquid," Journal of Atmospheric Science, 22:541-558, 1965.

${ }^{3}$ Lorenz, Edward N., "A Proposed Explanation for the Existence of Two Regimes of Flow in a Rotating Symmetrically-Heated Cylindricl Vessel," Proceedings of the First Symposium on the Use of the Models in Geophysical Fluids, $73-80,1956$.

"Williams, G. P., "Thermal Convection in a Rotating fluid Annulus: Part 1. The Basic Axisymmetric Flow," Journal of Atmospheric Science, 24:144-161, 1967a.

${ }^{5}$ Williams, G. P., "Thermal Convection in a Rotating Fluid Annulus: Part 2.," Journal of Atmospheric Science, 24:162-174, 1967b.

${ }^{6}$ Miller, T. L. and R. L. Gall, "R. L. Gall, "A Linear Analysis of the Transition Curve for the Baroclinic Annulus," Accepted for publication in the Journal of Atmospheric Science. 
7Hart, J. E.. "Studies of Earth Simulation Experiments," NASA Contractor Report NASA $C R-2753,1976$.

BMacaraeg, M. G., "A Mixed Pseudospectral/ Finite Difference Method for the Axisymmetric Flow in a Heated, Rotating Spherical Shell;" accepted for publication in the Journal of Computational Physics, 1985.

${ }^{9}$ Hussaini, M. Y., Street, C. L. and Zang, T. A. "Spectrai Methods for Partial Differential Equations," Conference on Applied Mathematics and Computing ARO Report 84-1.

10 Byers, H. R., General Meterology, New York: McGraw-Hill Book Company, Inc., 1959.

Table 1. Heat Transfer Ratios for Increasing Grashof and Ekman Numbers.

\begin{tabular}{|c|c|c|c|c|c|c|c|c|}
\hline Case & $G r$ & & Ek & GrEk 2 & $\frac{q_{\pi / 4}}{q^{(\pi / 4) C^{*}}}$ & $\frac{q_{\pi / 2}}{q_{(\pi / 2) C}}$ & $\frac{q_{5 c m}}{q_{5 c}}$ & $\frac{{ }^{q_{6 \mathrm{~cm}}}}{q_{6 c}}$ \\
\hline II & $\begin{array}{l}2.84 \times 10^{3} \\
2.84 \times 10^{4} \\
2.84 \times 10^{5} \\
2.84 \times 10^{6}\end{array}$ & $\begin{array}{l}2.78 \\
2.78 \\
2.78 \\
2.78\end{array}$ & $\begin{array}{ll}\times & 10^{-1} \\
\times & 10^{-2} \\
\times & 10^{-3} \\
\times & 10^{-4}\end{array}$ & $\begin{array}{c}219.2 \\
21.92 \\
2.192 \\
0.2192\end{array}$ & $\begin{array}{r}1.0 \\
2.0 \\
8.8 \\
15.8\end{array}$ & $\begin{array}{l}0.0 \\
0.0 \\
0.0 \\
0.0\end{array}$ & $\begin{array}{l}1.0 \\
0.861 \\
0.566 \\
0.287\end{array}$ & $\begin{array}{l}1.0 \\
0.913 \\
0.890 \\
0.898\end{array}$ \\
\hline
\end{tabular}

* $q_{c}=$ hedt transfer from conduction solution

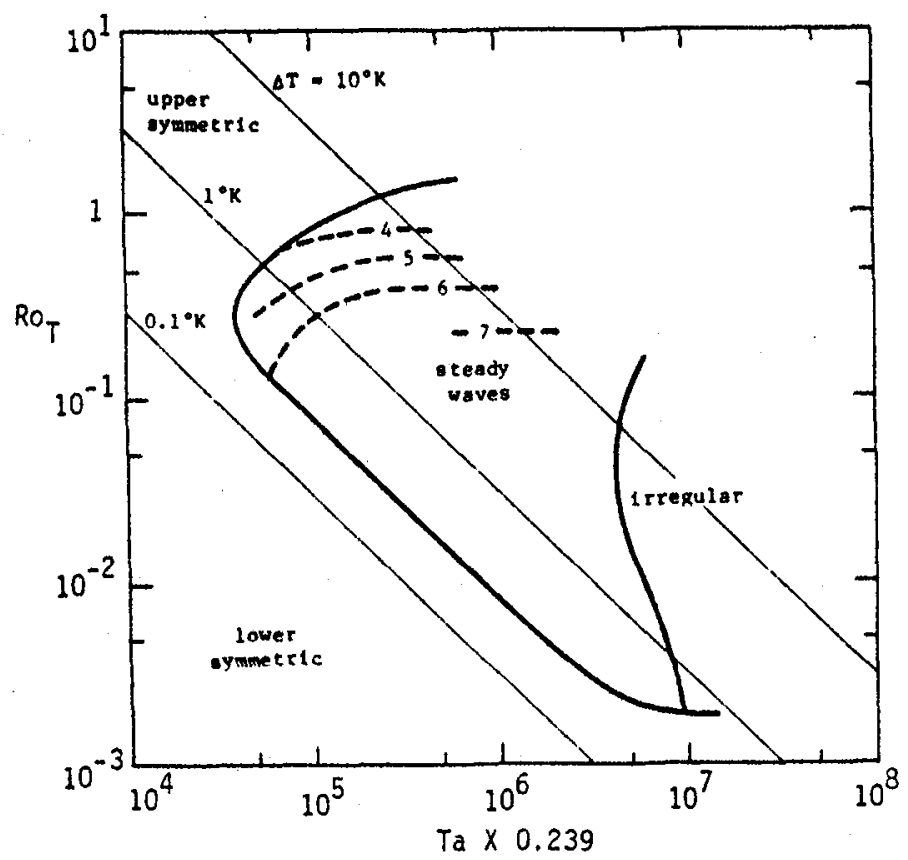

Fig. 1. Cylindrical Annulus Regime Diagram. 
top view:
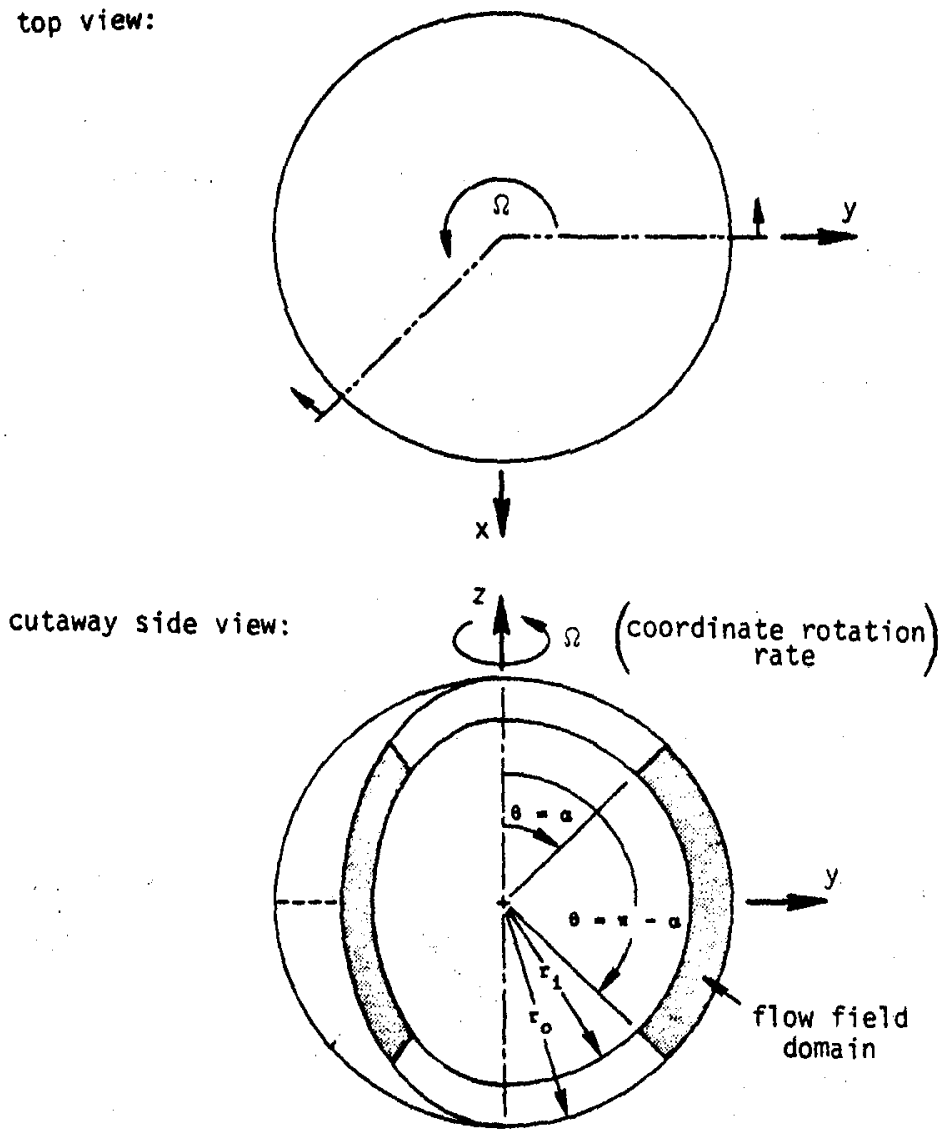

Fig. 2. Cutaway View of Physical Model.

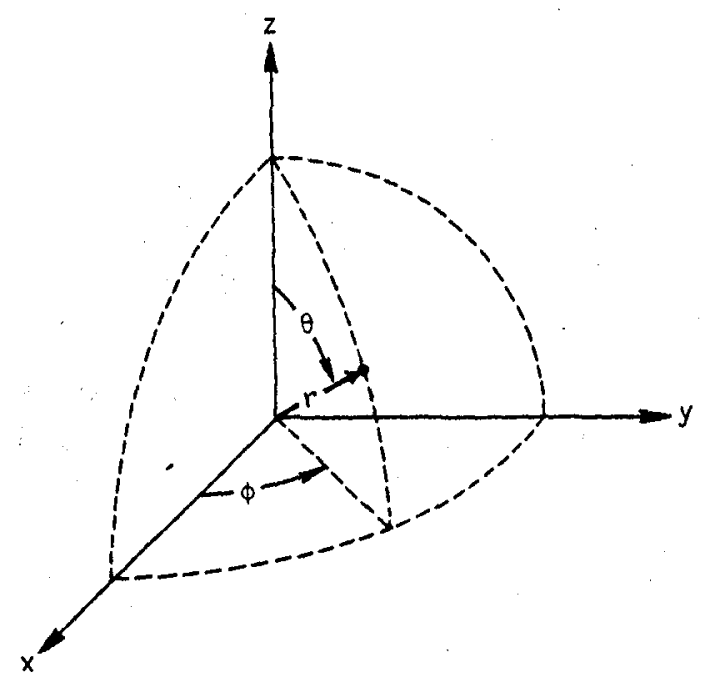

Fig. 3. The Spherical Coordinate System. 

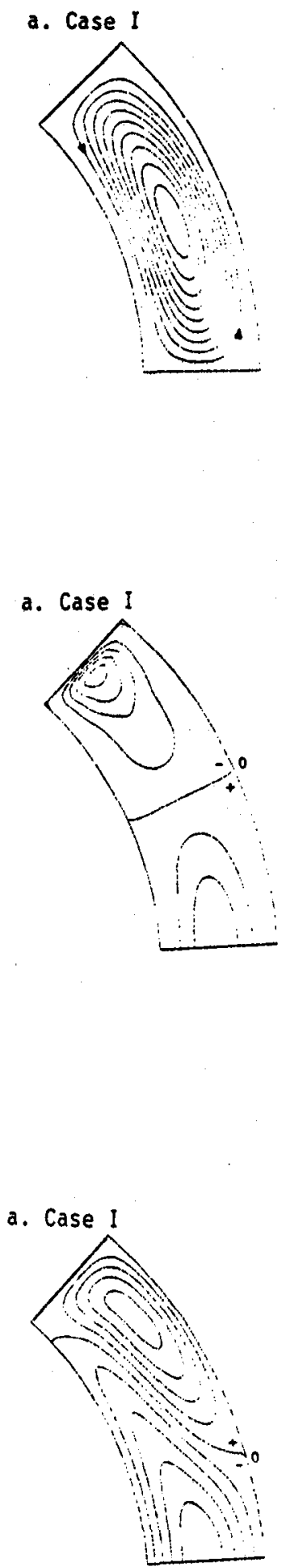
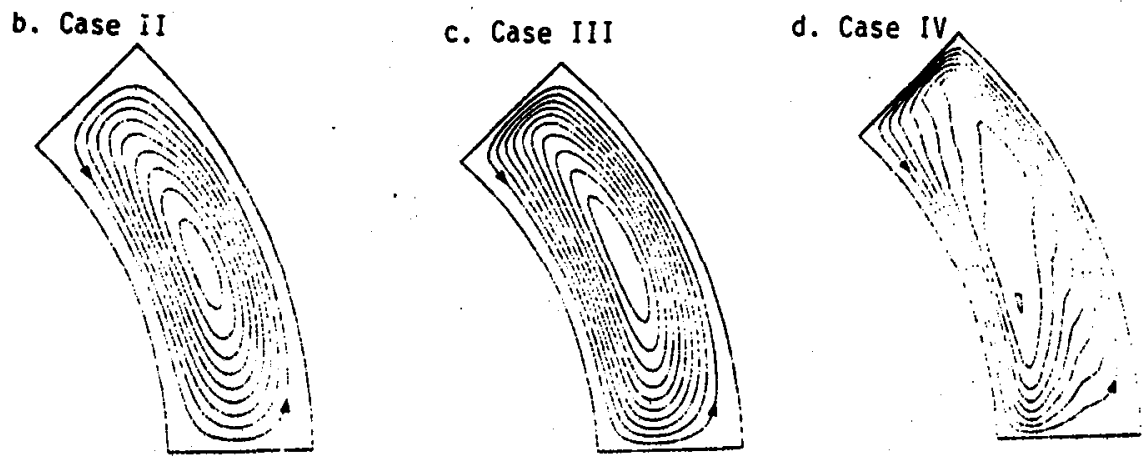

Fig. 4. $\psi$ Contour Plots for Cases I to IV.
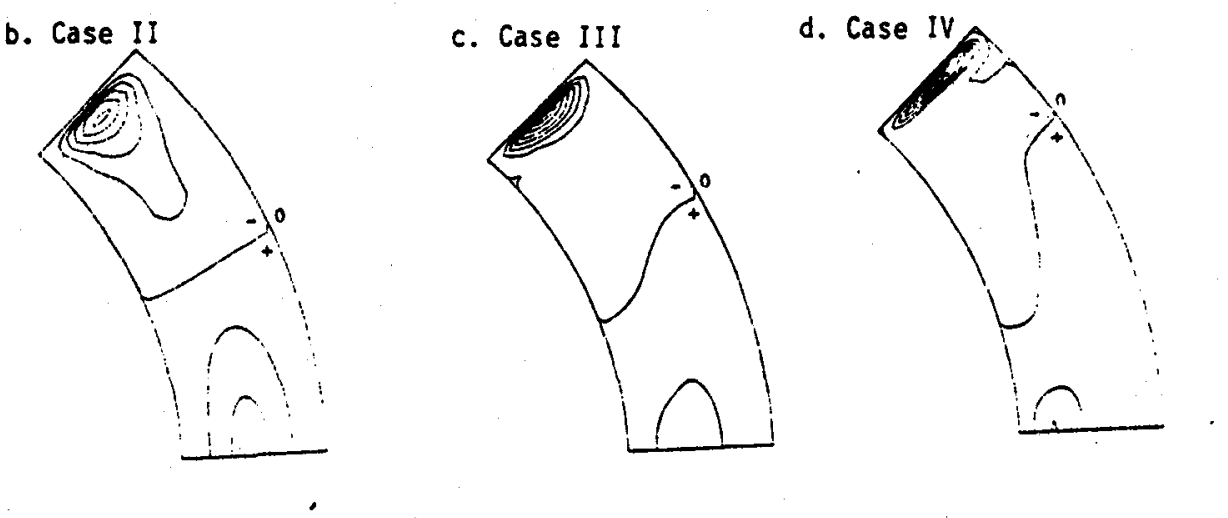

Fig. 5. w Contour Plots for Cases I to IV.

b. Case II

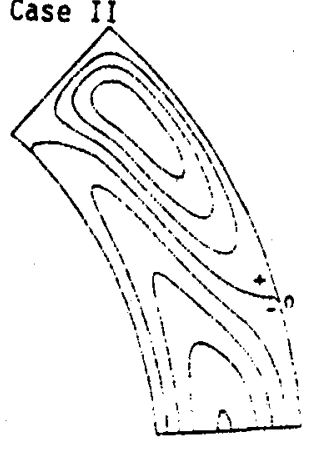

c. Case III

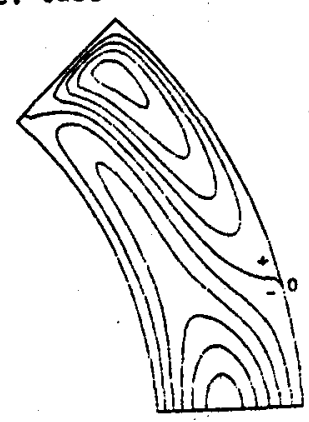

d. Case IV

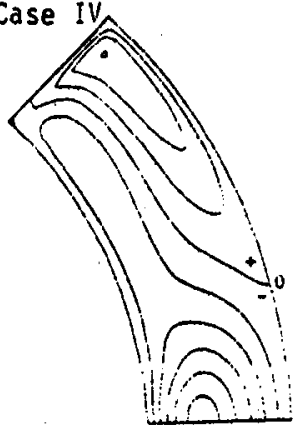

Fig. 6. u Contour Plots for Cases i to IV.

7 

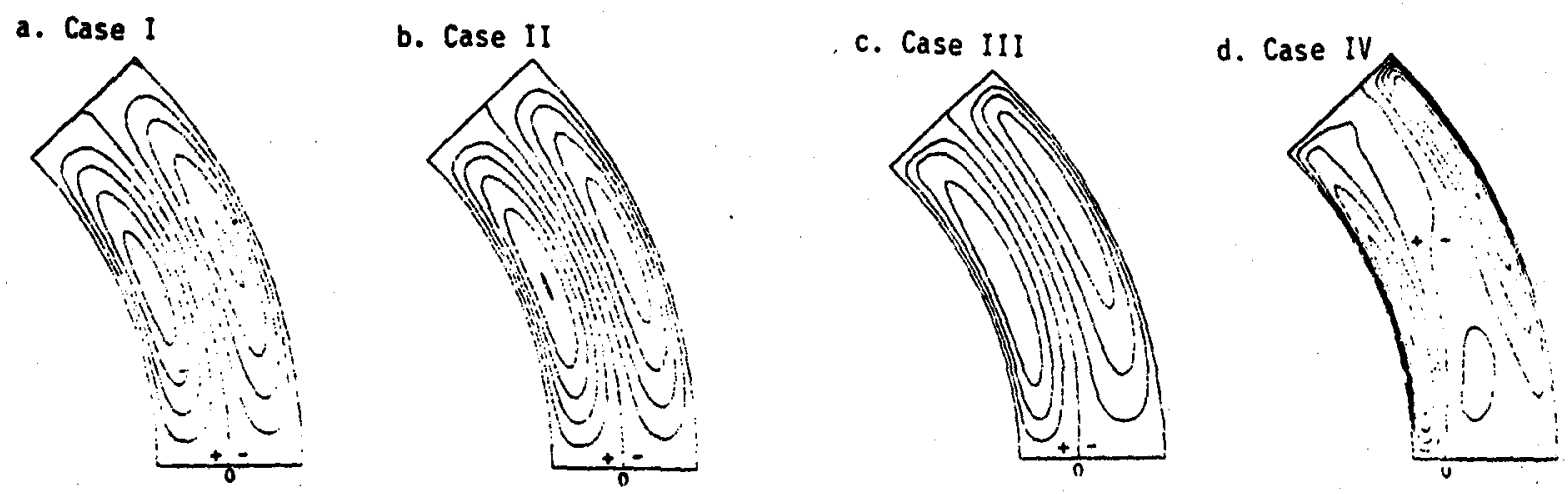

Fig. 7. $v$ Contcur Plots for Cases I to IV.
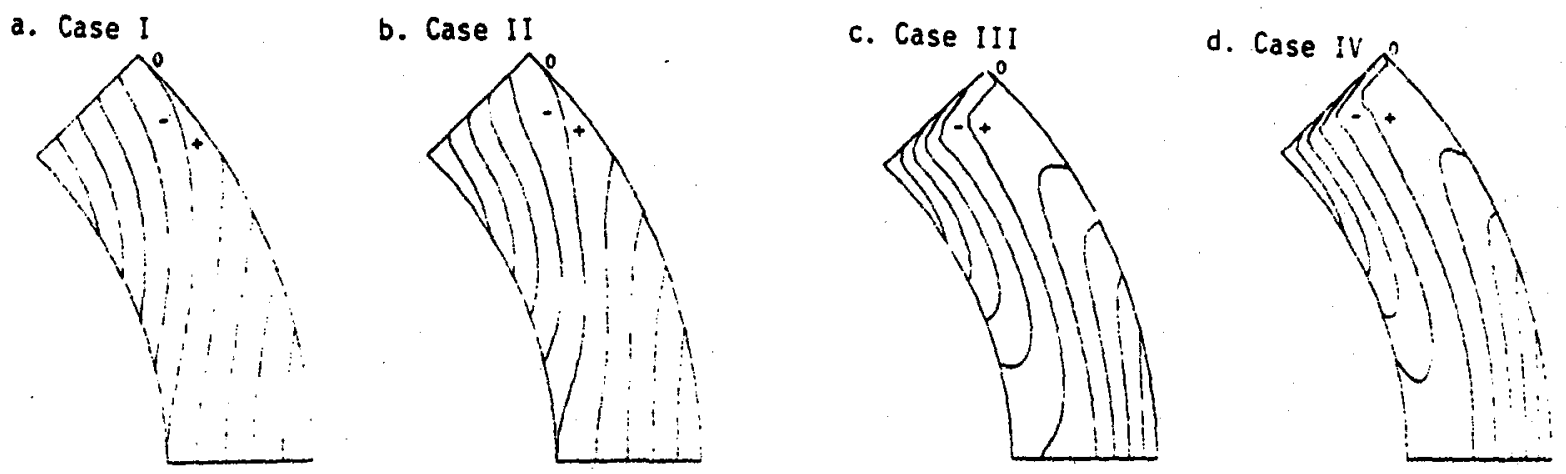

Fig. 8. T Contour Plots for Cases I to IV.

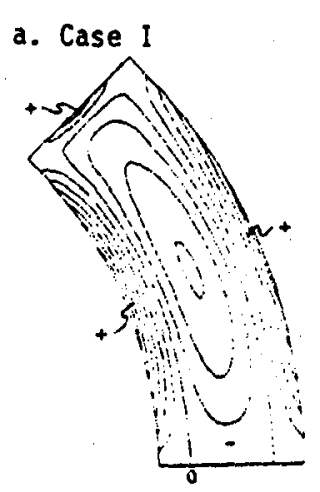

b. Casé II

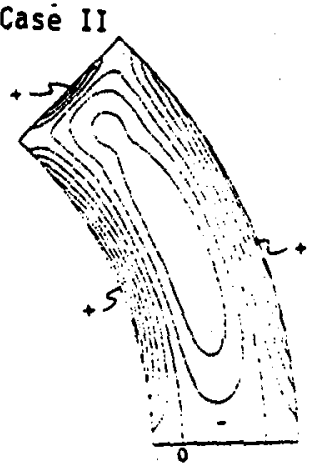

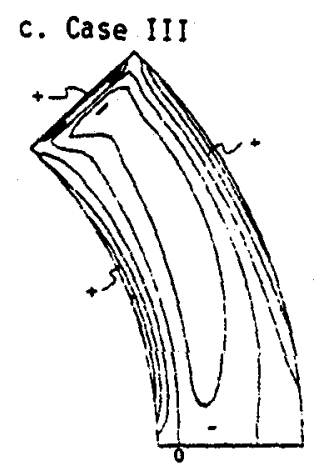

d. Case IV

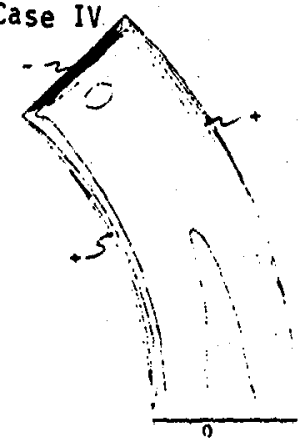

Fig. 9. E Contour Plots for Cases I to IV.

a. $\xi_{\max }=.00041 \mathrm{~s}^{-1}$ at $1.178 \mathrm{rad}, 5 \mathrm{~cm}$.

b. $\xi_{\max }=.0036 \mathrm{~s}^{-1}$ at $1.178 \mathrm{rad}, 5 \mathrm{~cm}$.

c. $\xi_{\max }^{\max }=.324 \mathrm{~s}^{-1}$ at $.7854 \mathrm{rad}, 5.5$

d. $\xi_{\max }^{\max }=1.719 \mathrm{~s}^{-1}$ at $.7854 \mathrm{rad}, 5.617 \mathrm{~cm}$. 



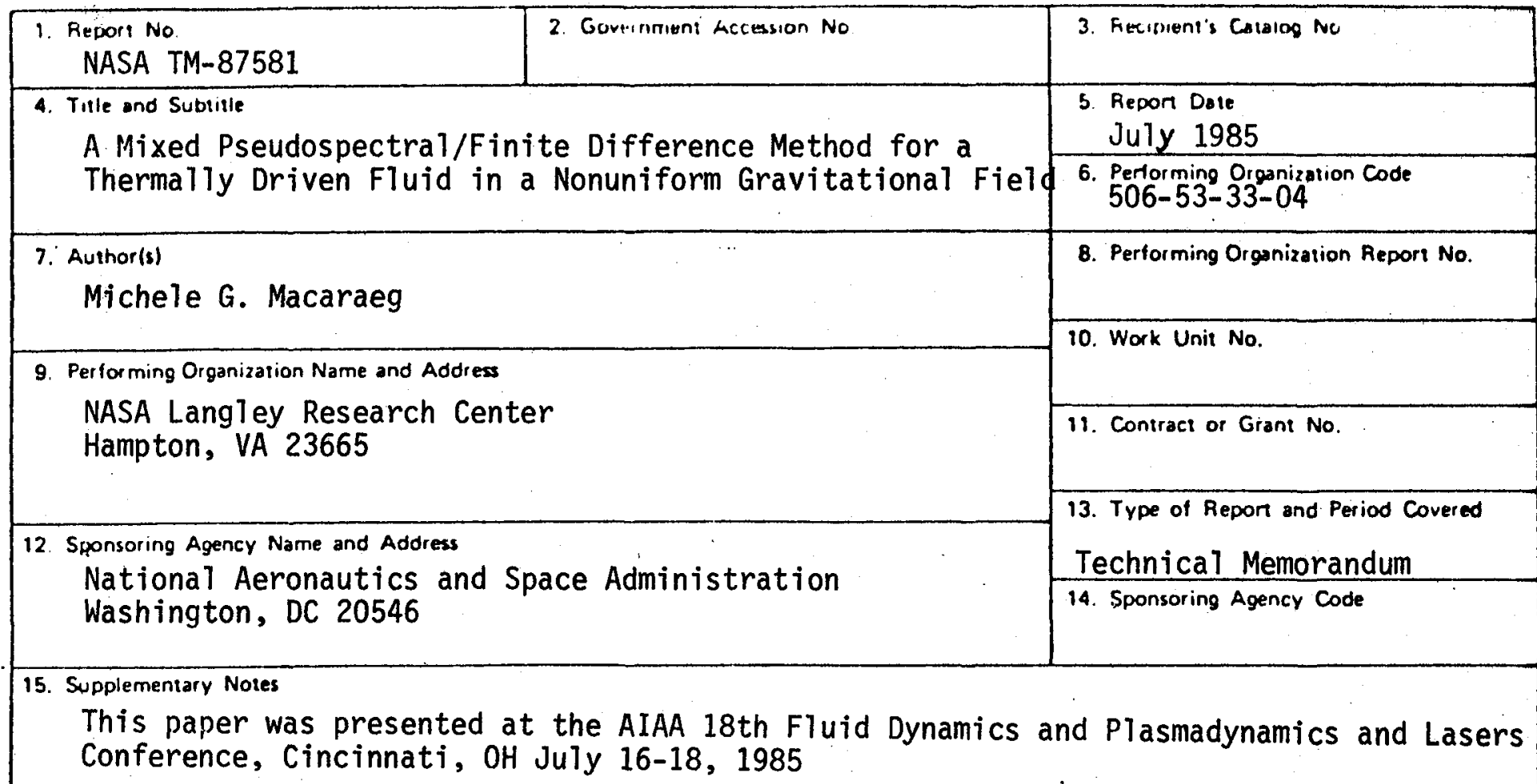

16. Abstract

A numerical study of the steady, axisymmetric flow in a heated, rotating spherical shel1 is conducted to model the Atmospheric General Circulation Experiment (AGCE) proposed to run aboard a later shuttle mission. The AGCE will consist of concentric rotating spheres confining a dielectric fluid. By imposing a dielectric field across the fluid a radial body force will be created. The numerical solution technique is based on the incompressible Navier-Stokes equations. In the method a pseudospectral technique is based on the incompressible Navier-Stokes equations. In the method a pseudospectral technique is used in the latitudinal direction, and a second-order accurate finite difference scheme discretizes time and radial derivatives. This paper discusses the development and performance of this numerical scheme for the AGCE which has been modelled in the past only by pure FD formulations. In addition, previous models have not investigated the effect of using a dielectric force to simulate terrestrial gravity. The effect of this dielectric force on the flow field is investigated as well as a parameter study of varying rotation rates and boundary temperatures. Among the effects noted are treproduction of larger velocities and enhanced reversals of radial temperature gradients for a body force generated by the electric field.

17. Key Words (Suggested by Author(s))

Axisymmetric flow

Pseudospectral/Finite
18. Distribution Statement

Unclassified-Unlimited

Subject Category 34

\begin{tabular}{|l|l|c|c|}
\hline $\begin{array}{l}\text { 19. Security Cassif. lof this report) } \\
\text { Unclassified }\end{array}$ & $\begin{array}{l}\text { 20. Security Classif. lof this pagel } \\
\text { Unclassified }\end{array}$ & $\begin{array}{c}\text { 21. No. of Pages } \\
9\end{array}$ & $\begin{array}{l}\text { 22. Price } \\
A 02\end{array}$ \\
\hline
\end{tabular}



